\title{
Full-Field X-ray Fluorescence Microscopy Using a Color $\mathrm{X}$-ray Camera
}

\section{Ursula Elisabeth Adriane Fittschen, ${ }^{1 *}$ Oliver Scharf, ${ }^{2}$ and Magnus Menzel ${ }^{3}$}

${ }^{1}$ Washington State University, Chemistry Department, Pullman WA 99164-4630

${ }^{2}$ IfG-Institute for Scientific Instruments GmbH, Rudower Chaussee 29/31, 12489 Berlin, Germany

${ }^{3}$ University of Hamburg, Institute for Applied and Inorganic Chemistry, Martin-Luther-King Plz. 6, 20163 Hamburg, Germany

*ursula.fittschen@wsu.edu

\section{Introduction}

Elemental imaging of several elements simultaneously and with detection limits in the ppb range is achieved by synchrotron-based X-ray fluorescence microscopy, also often referred to as micro-X-ray fluorescence (MXRF). This has been shown, for example, by imaging $\mathrm{Cu}$ and $\mathrm{U}$ distribution in contaminated sediments [1] and $\mathrm{P}, \mathrm{Ca}$, and Zn distribution imaging of single cells and mitochondria [2]. A review on environmental application can be found in reference [3]. XRF micro-probes are available at synchrotrons all around the world and allow for 2D imaging with spatial resolution from several micrometers down to the nanometer range $(30-100 \mathrm{~nm})$; the latter mainly at thirdgeneration synchrotrons.

$\mathrm{X}$-ray fluorescence (XRF). Interaction of X-rays with matter is in general dominated by the absorption of photons to generate photoelectrons. Because of the relatively high energy of X-ray photons, core shell electrons are often targeted by this process. Relaxation of the core hole occurs by a transition of an outer shell electron and emission of the transition energy as either an Auger electron or a photon, usually in the X-ray energy range. The emitted fluorescent $\mathrm{X}$-ray photon is characteristic of the excited element. This process is the basis for qualitative and quantitative determination of the elements present in the specimen, as well as XRF microscopy. As in other types of microscopy, MXRF can be performed in scanning mode or in full-field mode (Figure 1).

Full-field MXRF. In the full-field MXRF mode, the full sample is illuminated by the $\mathrm{X}$ rays from the source, and the fluorescence is guided by an optic to the fluorescence array detector. This is illustrated in Figure 1a. Horizontal and vertical slit systems can be used to shape the beam. However, most MXRF setups operate in scanning mode, which means the sample is moved through a focused primary X-ray beam that excites the fluorescent $\mathrm{X}$ rays. A single element fluorescence detector can be used. This is illustrated in Figure 1b. The scanning mode comes with disadvantages regarding in situ applications where the sample must remain fairly static or where the sample is brittle or in other ways sensitive to movements.

Here full-field MXRF is advantageous. An example is the imaging of elemental distributions in droplets $(10-20 \mu \mathrm{L}$ containing $\mathrm{Mn}, \mathrm{Ni}, \mathrm{Cu}$, and $\mathrm{Sc}$ ) while drying. This is shown in Figure 2. The droplets were allowed to dry undisturbed while the elemental information was recorded. Full-field MXRF allows for fast imaging of large areas (for example, $12 \times 12 \mathrm{~mm}^{2}$ at 1,000 frames per second and $264 \times 264$ pixels) and therefore simultaneous detection of elemental changes over the entire field of view, which can be important for certain in situ applications. However, the detectability of each element will depend on the fluorescence yield of the element and the total counts acquired. Thus, the recording frequency will be limited by the need to acquire enough counts for detecting specific elements. Full-field MXRF also allows fast 3D elemental imaging by taking images at different depths of the sample using a sheet beam.

\section{Materials and Methods}

Energy-sensitive camera/detector. Full-field MXRF in the past has suffered from low spectral (energy resolution) and low sensitivity, which were in part caused by the event processing and the low quantum efficiency of the array detectors used, as well as by optics with low transmission for fluorescent X-ray photons. Recently full-field MXRF has become significantly more powerful by the use of a two-dimensional energy-sensing camera/detector, increasing the sensitive thickness of the

(a)

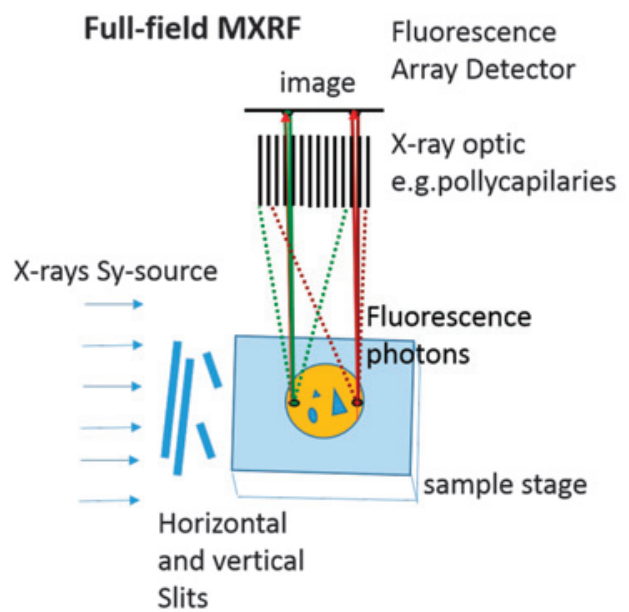

Figure 1: (a) Schematic of a typical full-field MXRF setup. The entire sample is illuminated at once, and the spatially resolved elemental information is obtained by an array detector combined with a suitable optic. (b) Schematic of a typical scanning MXRF setup. The sample is scanned through a focused beam. The primary beam is usually at $45^{\circ}$ to the sample surface. 


\section{TAKKEA F日ESH \\ $A P P \cap \square A \subset-H \square S E M$}

Intuitive multi-touch screen interface • High spatial resolution • Unprecedented analytical versatility Easy navigation and operation • Fast imaging and data acquisition

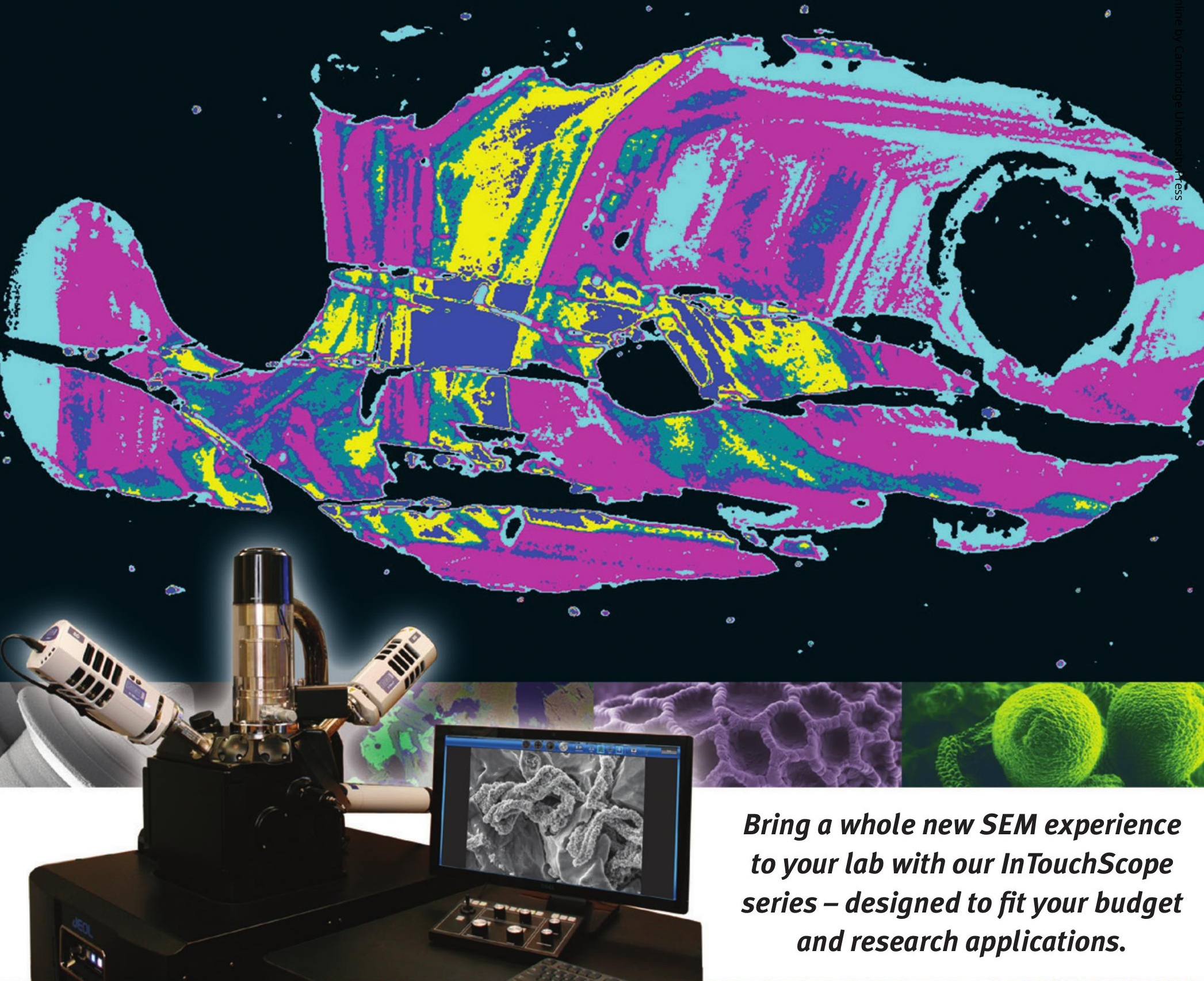

पis $>$

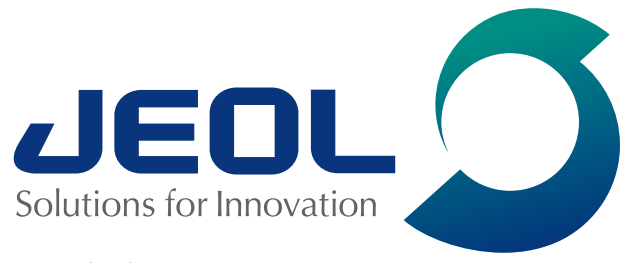

www.jeolusa.com/fresh 
Electron Microscopy Sciences

P.O. Box $550 \bullet 1560$ Industry Rd. • Hatfield, Pa 19440

Tel: (215) 412-8400 • Fax: (215) 412-8450

email: sgkcck@aol.com or stacie@ems-secure.com

look for us... YouTube if

\section{B. Flat ${ }^{\text {TM }}$ Holey Garbon Grids for Gryo-TEM}

C-flat TM is an ultra-flat, holey carbon-coated TEM support grid for transmission electron microscopy (TEM). Unlike competing holey carbon films, C-flat ${ }^{\mathrm{TM}}$ is manufactured without plastics, so it is clean upon arrival and the user has no residue to contend with.

\section{C-flat ${ }^{\text {TM }}$ leads to better data sets.}

Made with patent pending technology, $\mathrm{C}$-flat ${ }^{\mathrm{TM}}$ provides an ultra-flat surface that results in better particle dispersion and more uniform ice thickness. Patterning is done using deep-UV projection lithography, ensuring the most accurate and consistent hole shapes and sizes down to submicron features. The precise methods by which $\mathrm{C}$-flat ${ }^{\mathrm{TM}}$ is manufactured eliminate artifacts such as excess carbon and edges around holes.

\section{G-filat ${ }^{\text {TM }}$ is affordable}

C-flat ${ }^{\mathrm{TM}}$ is available in 25,50 , and 100 packs at a per-grid price less than competing products.

\section{C-flat ${ }^{\mathrm{m}}$ Customization}

We realize that each customer has unique needs since specimens vary greatly in composition and size. To meet the diverse and demanding needs of the cryoTEM community, C-flat ${ }^{\mathrm{TM}}$ can be customized to meet a user's specific requirements.

Please contact EMS with any custom C-flat ${ }^{\mathrm{TM}}$ requests. We will be glad to provide you with a quote for specialized C-flat ${ }^{\mathrm{TM}}$ grids. Requests for customized parts can be made directly to EMS via e-mail to sgkcck@aol.com

C-Flat ${ }^{\mathrm{TM}}$ is now available in the standard version and a new thick version that doubles the carbon thickness from approximately $20 \mathrm{~nm}$ to $40 \mathrm{~nm}$.

\section{DuraSfI' ${ }^{\mathrm{m}}$ Film and Mesh for TEM}

DuraSiN ${ }^{\mathrm{TM}}$ Film and Mesh products are affordably-priced, durable, nonorganic, low scatter support grids for quantitative TEM and X-ray analysis. DuraSiN ${ }^{\mathrm{TM}}$ products are made of a thin, high quality, low-stress silicon nitride membrane supported around its perimeter by a rigid silicon substrate.

Unlike other support films and grids, DuraSiN TM Film and Mesh products can withstand harsh chemical and temperature environments. For example, DuraSiN ${ }^{\mathrm{TM}}$ Film or Mesh products could be used as a substrate onto which nanowires could be directly grown from a strong acidic solution. Once the nanowires are grown, the specimen is immediately ready for imaging and analysis in the TEM. With direct deposition, no longer will you have to prepare a sample on one substrate only to then have to transfer it to a support grid for imaging.

\section{Applications:}

\section{SEMICONDUGTORS}

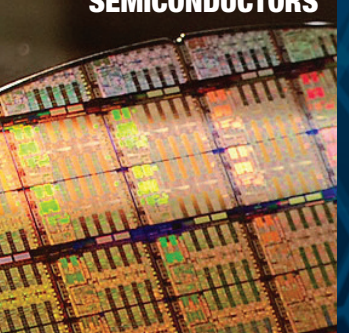

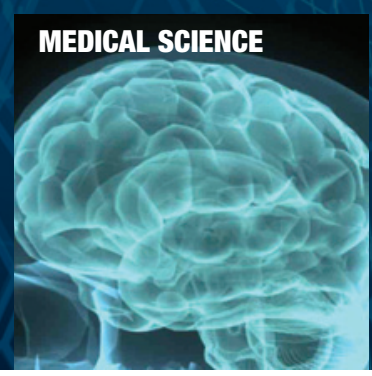

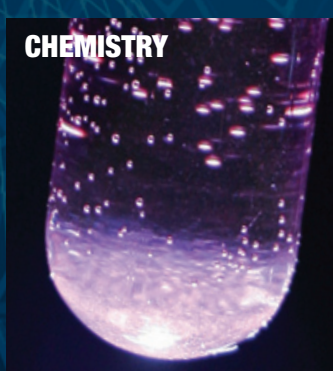

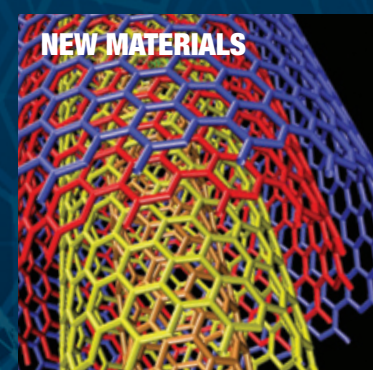
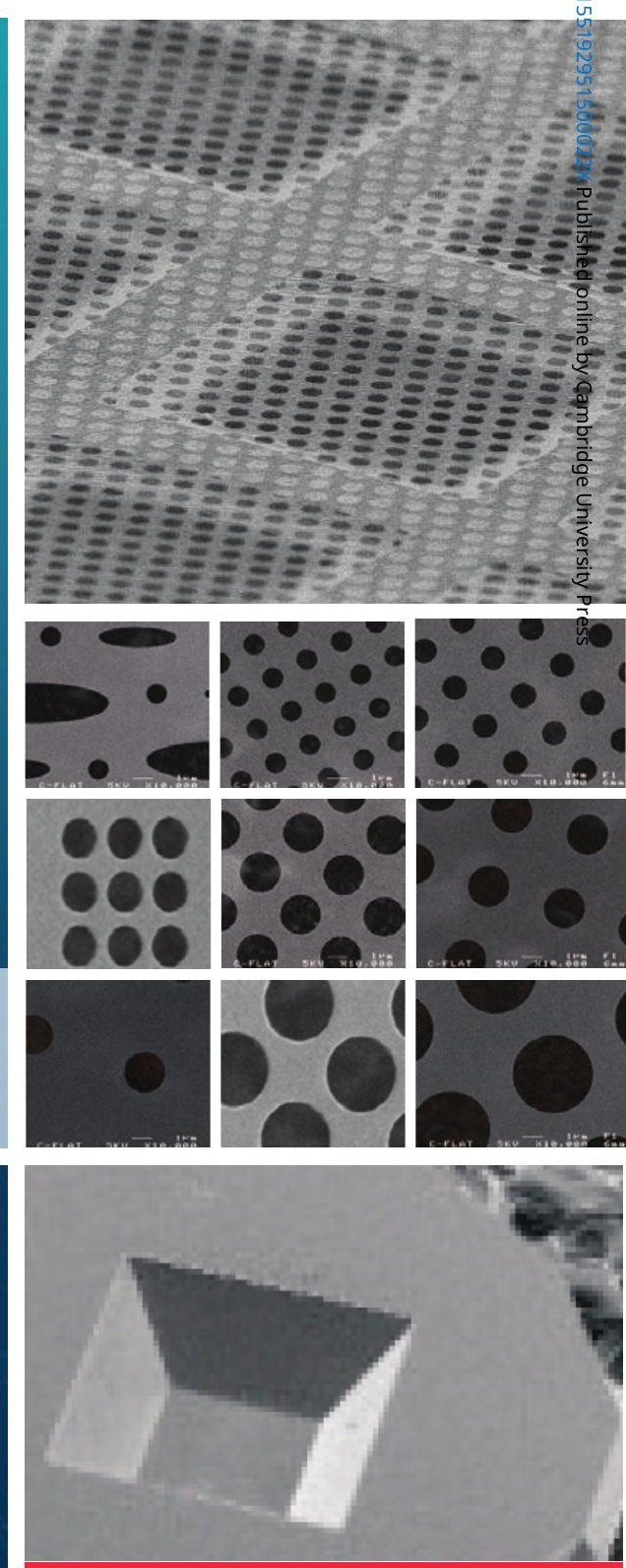

SEM image of a DuraSiN ${ }^{\text {Tm }}$ Film

(taken from the back side)

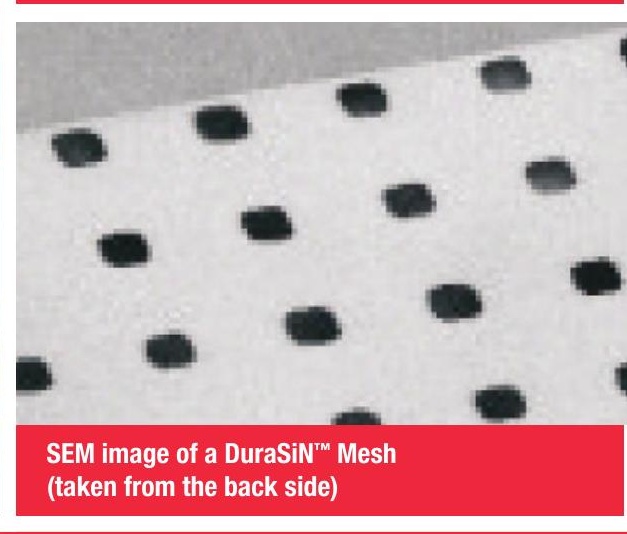

\section{Electron}

Microscopy Sciences 


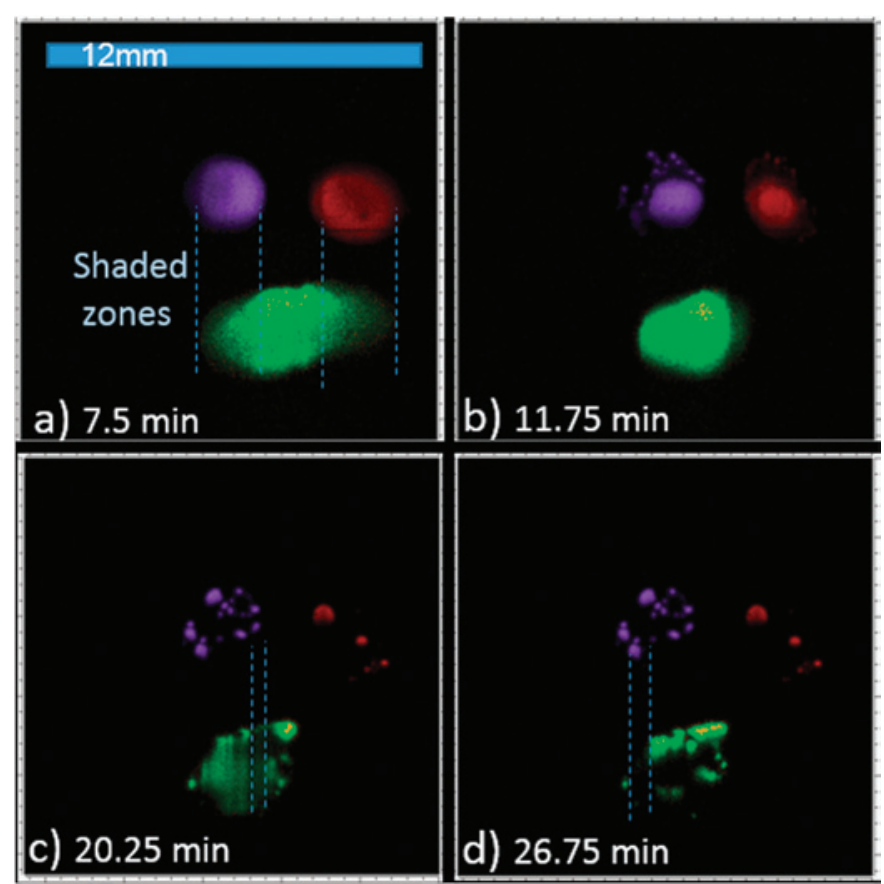

Figure 2: Elemental images collected from droplets while drying using an X-ray color camera in TXRF illumination geometry. The droplet consists of two $10 \mu \mathrm{L}$ droplets and one $20 \mu \mathrm{L}$ droplet: $\mathrm{Mn}$ (violet), $\mathrm{Ni}$ (red), and $\mathrm{Cu}+\mathrm{Sc}$ (green). The X-ray source is located at the top of each picture (shaded regions are indicated by dotted lines). (a) Image taken after $7.5 \mathrm{~min}$ drying, (b) image taken after $11.75 \mathrm{~min}$ of drying, (c) image taken after $20.5 \mathrm{~min}$, and (d) image of completely dried specimen after 27 min. Measurements were carried out at the Beamline/BESSYII instrument at Helmholtz-Zentrum Berlin.

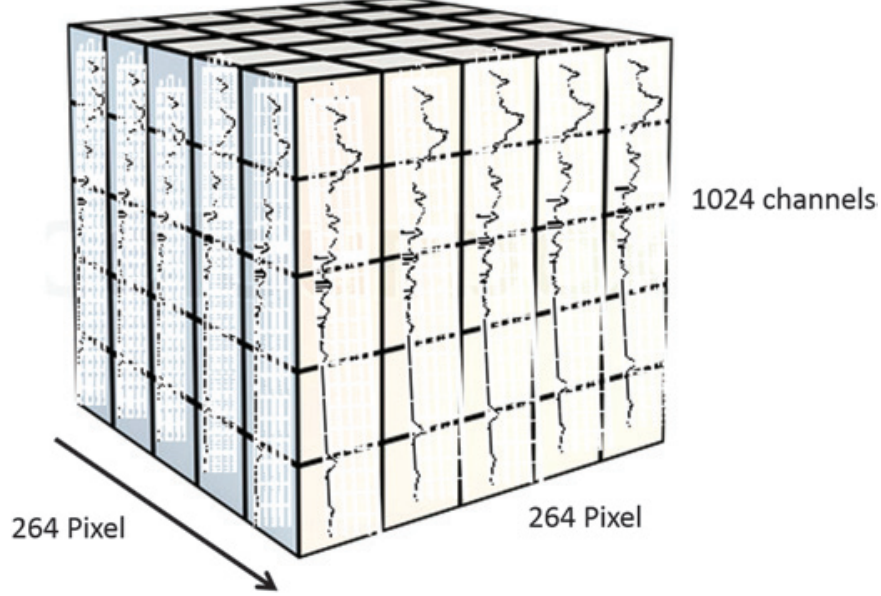

Figure 3: Schematic showing multiple dimensions in the acquisition of an XRF spectrum at each pixel. Similar data cubes may be acquired in both scanning and full-field microscopy modes. Reprinted with permission from Oliver Scharf.

detector, using arrays of capillary optics ( $>200 \mathrm{k}$ capillaries) to guide more fluorescence photons to the detector array, and improving event processing software.

Modern full-field array detectors like the SLcam ${ }^{\circledR}$ provide spectral (energy) resolution of $<160 \mathrm{eV}$ [4], comparable to single-chip silicon drift detectors (SDDs). At each pixel a full spectrum comprising an energy range of $>10 \mathrm{keV}$ is acquired. Figure 3 shows the typical data cube for a spectrum image with a $264 \times 264$ array and spectra collected over 1,024

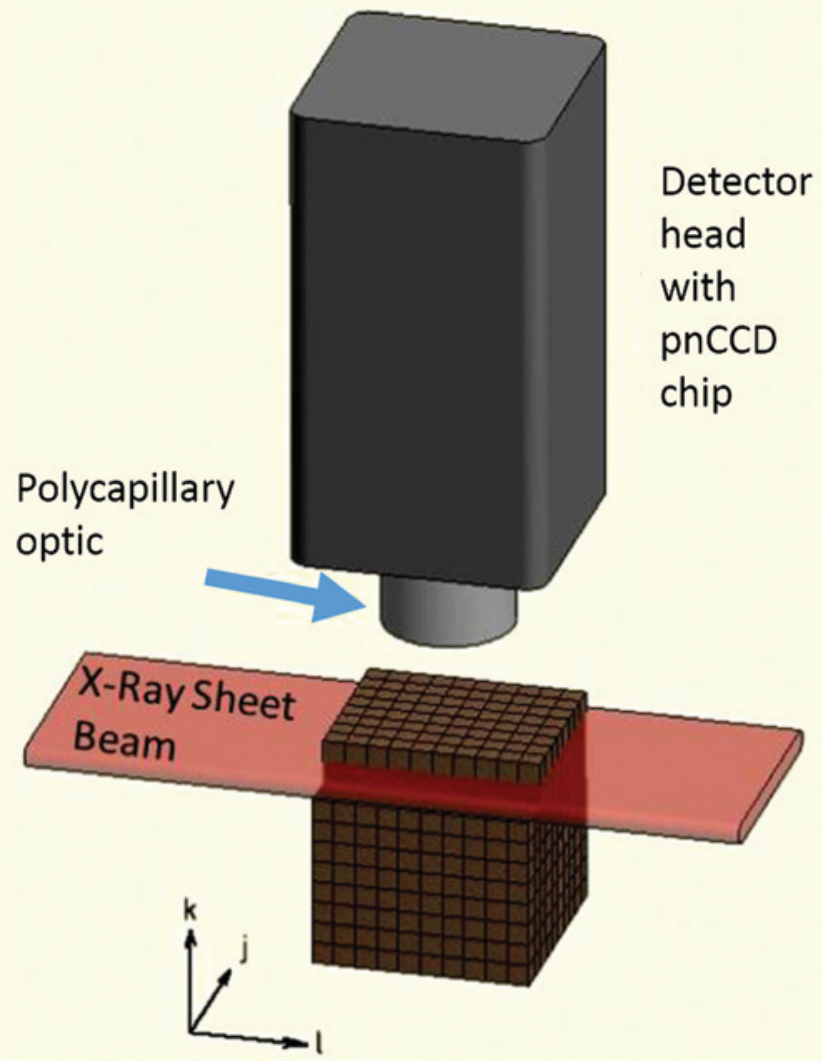

Figure 4: Schematic showing a sheet beam exciting a slice of specimen and an energy-dispersive detector detecting elemental signals. Reprinted from [5] with permission from the Royal Chemical Society.

channels. The counts acquired for a specific element line can be extracted and displayed to show the spatial distribution of this element in an X-ray map. However, these array detectors do not achieve the high count rates of SDD detectors, for example, the $\mathrm{SLCam}^{\circledast}$ array camera count rates currently are only about $22 \mathrm{cps} /$ pixel. The spatial resolution obtained with full-field setups is in the single-digit micrometer range and is comparable to spatial resolutions achieved in scanning mode at second-generation synchrotron facilities.

Excitation and detection. In full-field X-ray microscopy, the sample is illuminated with a grazing incident beam, a total reflected beam, or a sheet-like beam $[5,6]$. A schematic of the setup using a sheet-like X-ray excitation beam from Radkte et al. [5] is shown in Figure 4. A 3D image of an object can be obtained by translating the object through the sheet beam, where the in-depth image resolution is given by the thickness of the sheet beam. Elemental specific XRF data can be obtained from this excitation. Semiconductor array detectors such as charge coupled devices (CCDs) are used to achieve an elemental analysis as the object passes through the sheet beam. CCDs are in general energy-dispersive; however, to achieve energy (spectral) resolution comparable to SDDs, sophisticated devices like the silicon-based SLcam ${ }^{\circledast}$ (developed by PNSensor GmbH, Munich, and IFG, Berlin, with other partners ) have to be used [4]. Such devices are often referred to as color X-ray cameras, or CXCs, because they provide energy resolution sufficient to discriminate 


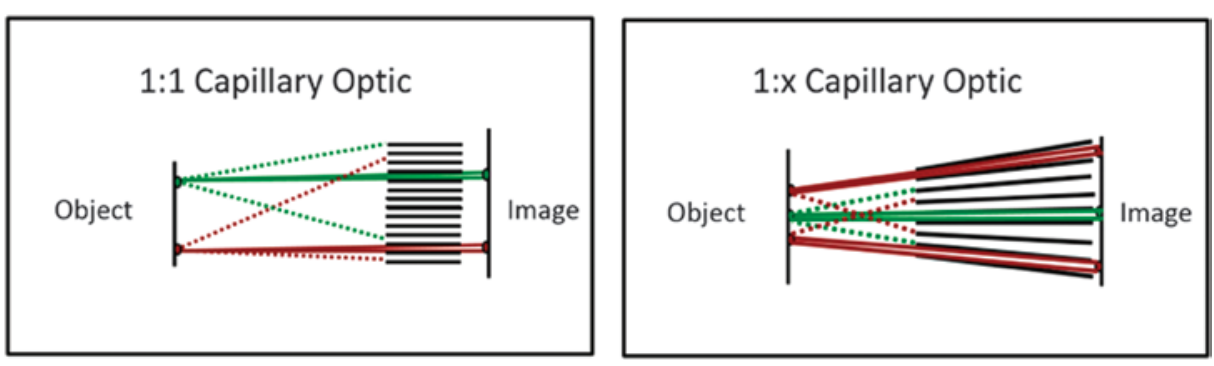

Figure 5: Schematic diagrams of a 1:1 capillary optic and a 1:x optic. Reprinted with permission from Oliver Scharf. spectrum from the front surface of the object, $\mathrm{Ca}$ and $\mathrm{Sr}$ are known to be from the ivory, $\mathrm{Cu}$ is likely a pigment that once decorated part of the design, and Fe could be either from a pigment outling the design or picked up from the burial sediments. These assumptions about the elements in the object were largely derived from the elemental images produced by the SLcam ${ }^{\circledast}$. The assumptions about the Fe distribution were gleaned from the elemental fluorescence lines (comparable to different colors in the light optical regime).

Full-field X-ray microscopy is more restricted than $\mathrm{X}$-ray absorption microscopy regarding the optics set between the array detector and the sample to be imaged [7]. This is because the optical setup needs to be fairly achromatic to guide photons of different energy accurately, which is necessary in MXRF. Pinholes have been successfully used $[8,9]$. However, polycapillary optics allow for very high transmission of $X$ rays of various energies. These optics may be used as guiding optics, achieving the resolution given by the array detector (Figure 5a), or they may project an enlarged image on the detector (Figure 5b). For example, the spatial resolution achieved with the SLcam ${ }^{\oplus}$ is about $50 \mu \mathrm{m}$ using a 1:1 optic, but it can be improved by enlarging the image to 1:5 and even 1:8 [4]. By using an algorithm it is possible to achieve sub-pixel resolution, better than $5 \mu \mathrm{m}[10]$.

\section{Results}

Drying of aqueous drops. A major advantage of full-field XRF imaging over scanning MXRF is rapid recognition of the major features in elemental distributions. The field of view is usually large, for example, $12 \times 12 \mathrm{~mm}$ [4], and therefore an overview of the sample and "non-targeted" results are obtained. Full-field observation is also favorable for imaging objects in situ, especially in environments where liquids are involved or when the specimen must remain static. Imaging of droplets while drying requires a non-destructive probe operating under ambient conditions. The experiment shown in Figure 2 could not have been accomplished in an electron microscope that typically must place the specimen under vacuum. That experiment also had a temporal aspect. While XRF images were acquired every 15 seconds, only a selection of images taken over the 27-min drying process is displayed in Figure 2. Simultanous imaging of spatially separated areas can also be realized using a full-field setup, allowing the observation of process changes over time. Time-resolved measurements, however, are limited by the acquisition rate and count rate [11].

Ancient Phoenician object. A Phoenician ivory (eighthcentury BCE) from the Badisches Landesmuseum, Karlsruhe, Germany, was examined by full-field XRF imaging using synchrotron radiation, a straight polycapillary optic (Figure 5a), and the SLcam ${ }^{\circledR}$ energy-dispersive camera/detector by Reiche et al. [12]. This non-destructive analysis provided distributions of the major, minor, and trace elments on the surface of the carved object (Figure 6). Of the major elements in the global manner in which some Fe deposits were located in the deep crevices of the carving, whereas other Fe deposits appear to follow the the surface cracks. These elemental results help to produce a hypothesis concerning the colors employed in the original ancient artwork.

Shadowing effects. A static position of the sample is indispensable for diagnostics in total reflection X-ray fluorescence (TXRF) analysis. The term TXRF decribes a certain geometry in XRF elemental analysis that allows for trace element determination in minute amounts of a sample. In TXRF the excitation beam inpinges at a very small angle (in the range of $0.1^{\circ}$ ) onto the sample carrier surface. The shadowing of parts of the sample by rough surface features is an interference in TXRF, and better understanding of shadowing would improve the method significantly. Imaging of shadings in the TXRF geometry was possible using a full-field micro-XRF setup [6]. Figure 7 shows shadows in a $\mathrm{Cu}$ fluorescence image of a copper plate caused by roughness and particles as the plate was illuminated in total reflection excitation geometry from the bottom of the figure. The $\mathrm{Cu}$ image was captured using a color X-ray camera.

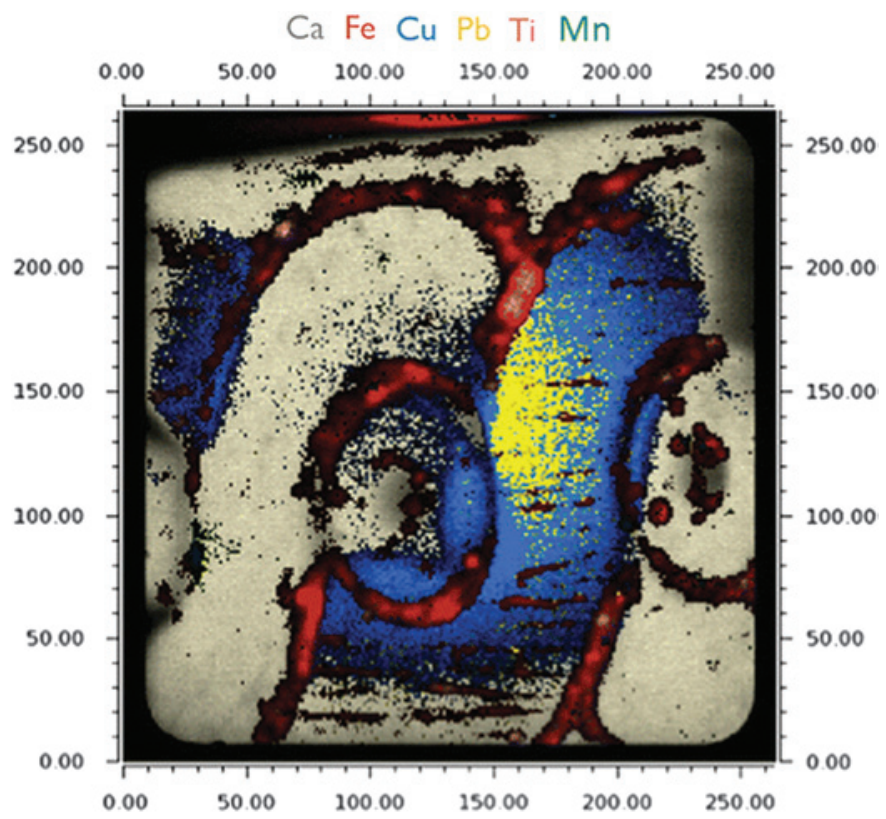

Figure 6: Analysis of an ancient Phoenician carved ivory object. Elemental images overlay of Ca K-alpha image (ivory), Cu K-alpha image (blue), Pb L-alpha image (yellow), Ti K-alpha image (purple), and Fe K-alpha image (red). Full width $=13 \mathrm{~mm}$. Reprinted from [12] with permission from the American Chemical Society. 


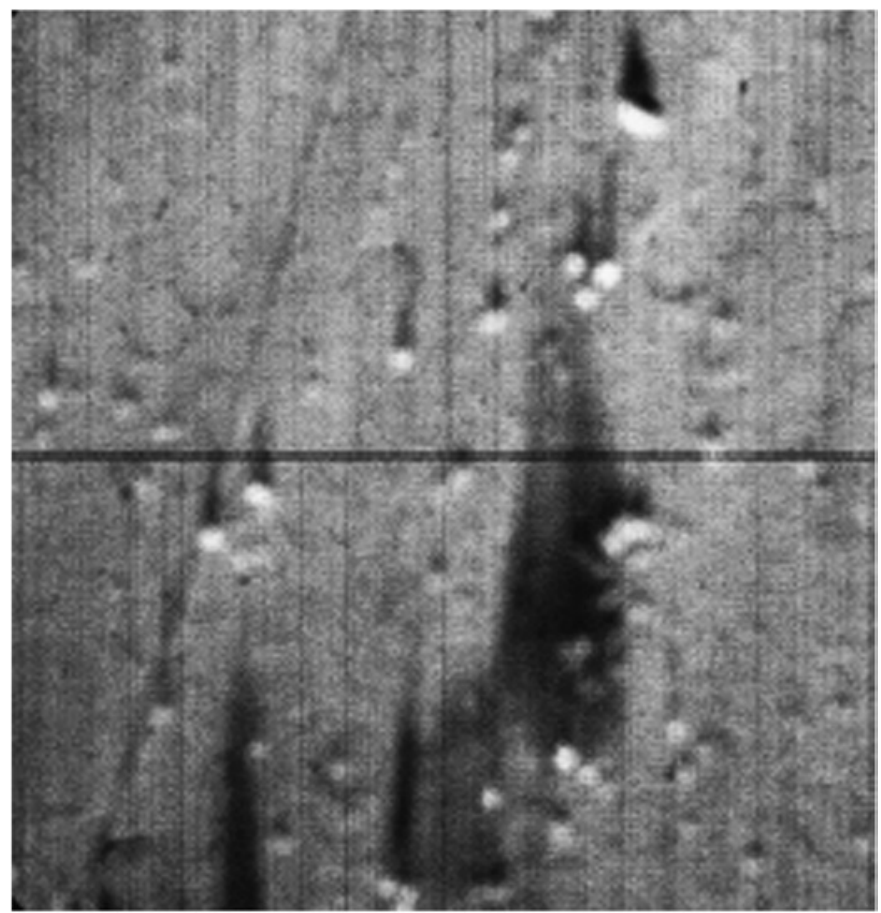

Figure 7: Shadows on a Cu plate caused by roughness and particles recorded in total reflection geometry with an SLcam ${ }^{\circledR}$ color X-ray camera. Reprinted from [6] with permission from Elsevier.

Shading is also observed in the drying droplets shown in Figure 2. Changing shading patterns (blue dotted lines) are caused by the changing physical shape of the specimen recorded in the drying experiments of droplets. Shadows are clearly visible, and they change in dimensions during the drying process.

Reconstructing 3D images from sheet-beam slices. Three-dimensional imaging can be achieved with full-field XRF by slicing the object with a sheet beam and reconstructing the image slices, a method introduced by Radtke et al. [5]. In general the synchrotron beam can be collimated into a sheet beam vertically and horizontally by slit systems. In their study two different geometries were tested. A sidewards positioning of the camera did not provide optimal flux on the sample. By positioning the camera horizontal looking down on the sample, higher flux was obtained because in such geometry the second multilayer of the double multilayer monochromator (DMM) can be bent to focus and generate an excitation beam of $50 \mu \mathrm{m}$ height. Figure 8 shows an example of a hornet imaged with a sheet beam. The specimen was chosen because insects are often used as biomonitors of metal contamination in the environment. An animation of the three-dimensional distributions in this image can be found in the supporting material of [5]. Data from 200 layers, corresponding to about $6 \mathrm{~ms}$ per voxel, were measured. The total measurement time was about 24 hours.

Absorption near-edge structure. In synchrotronbased full-field emission XANES microscopy, a narrow energy range on the excitation side, $\Delta \mathrm{E}$ less than $1 \mathrm{eV}$, may be achieved using crystal monochromators. The absorption near-edge (XANES) features of one selected elemental fluorescence line can be imaged. This type of analysis can provide information on the distribution of a specific species of an element because the near-edge fine structure changes for different chemical species of the regarded element. Although laboratory XANES point analysis has become quite powerful, XANES imaging suffers from low intensity of the analytical signal. Synchrotron sources can provide a small $\Delta \mathrm{E}$, efficient imaging optics, and now the color X-ray camera, SLcam ${ }^{\circledast}$. Full-field fluorescence mode micro-XANES was demonstrated recently by Tack et al. [13]. Figure 9 shows differential imaging of $\mathrm{Fe}^{0}$ and $\mathrm{Fe}^{3+}$ in an iron test sample containing both $\mathrm{Fe}$ foil and $\mathrm{Fe}_{2} \mathrm{O}_{3}$ powder.
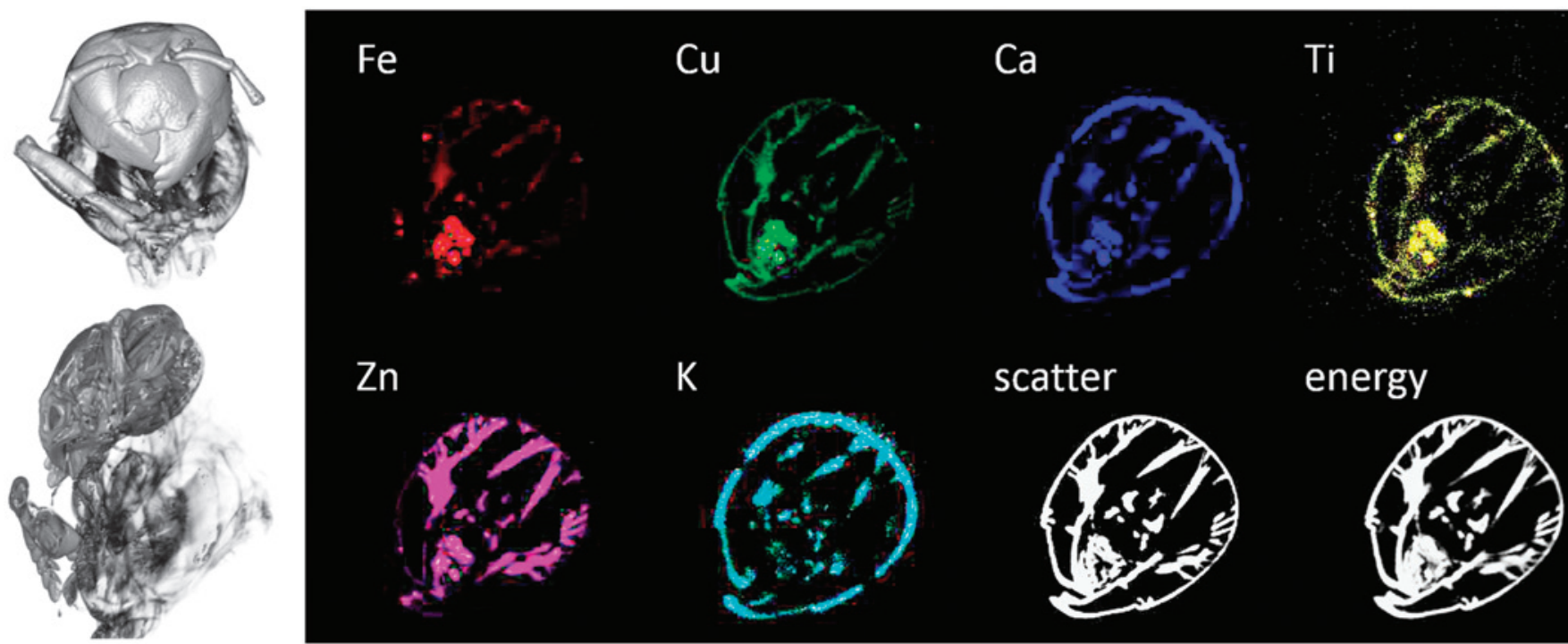

\section{$\mathrm{Zn}$}

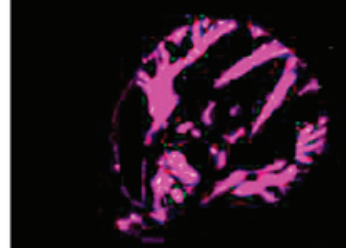

K

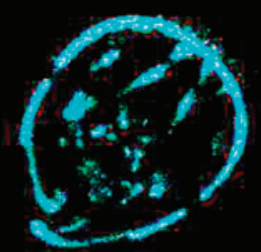

scatter

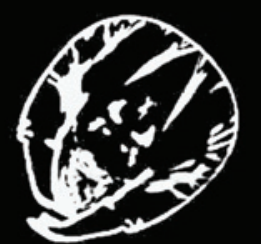

energy

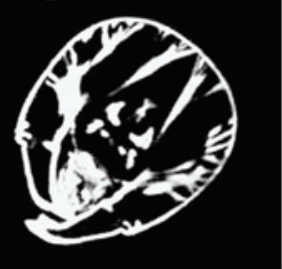

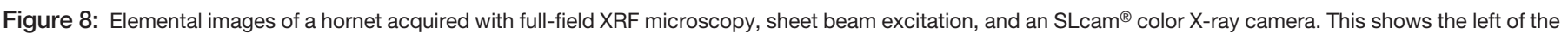

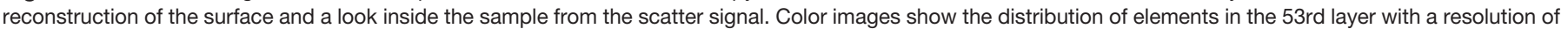

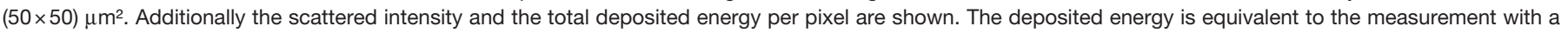
conventional CCD without energy resolution. Reprinted from [5] with permission from the Royal Chemical Society. 

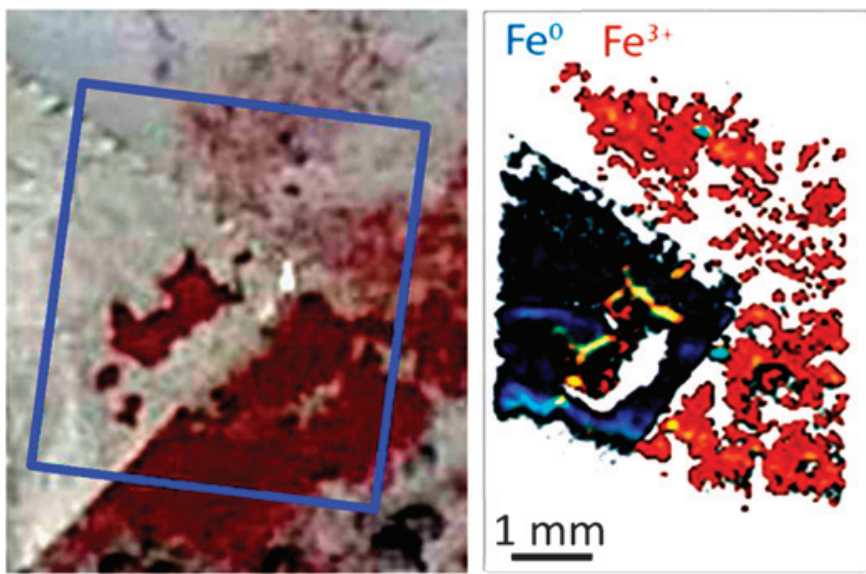

Figure 9: Left: Photograph of an iron test sample composed of a $4 \mu \mathrm{m}$ thick $\mathrm{Fe}$ foil, $\mathrm{FeO}$ particles, and $\mathrm{Fe}_{2} \mathrm{O}_{3}$ powder. The blue box shows the area that was investigated using the SLcam ${ }^{\circledR}$. Right: Result of differential imaging of the iron test sample after $20 \mathrm{~min}$ and monitoring the Fe K $\alpha$ signal at 7,120 and 7,143 eV. Spatial resolution $48 \times 48 \mu \mathrm{m}^{2}$. Reprinted from [13] with permission from the American Chemical Society.

\section{Conclusion}

Full-field XRF microscopy using a color X-ray camera is a powerful tool to quickly image large areas (up to about $100 \mathrm{~mm}^{2}$ ) with spatial resolution from $50 \mu \mathrm{m}$ to $5 \mu \mathrm{m}$. It is ideally suited to non-destructive study of fragile samples, such as those found in cultural heritage research, and for in situ imaging.

\section{Acknowledgment}

The authors thank Martin Radkte and Uwe Reinholz from Bamline at BESSY for discussions and the Helmholtz Zentrum Berlin (HZB) for the allocation of synchrotron radiation beamtime. Magnus Menzel and Ursula Fittschen thankfully acknowledge the financial support from HZB.

\section{References}

[1] DM Singer et al., Environ Sci Technol 43 (2009) 630-36.

[2] S Matsuyama et al., X-Ray Spectrom 38 (2009) 89-94.

[3] UEA Fittschen and G Falkenberg, Spectrochim Acta Part B 66 (2011) 567-80.

[4] O Scharf et al., Anal Chem 83 (2011) 2532-38.

[5] M Radtke et al., J Anal At Spectrom 29 (2014) 1339-44.

[6] UEA Fittschen et al., Spectrochim Acta Part B 99 (2014) $179-84$.

[7] UEA Fittschen and G Falkenberg, Anal Bioanal Chem 400 (2011) 1743-50.

[8] M Alfeld et al., AIP Conference Proceedings 1221 (2010) 111-18.

[9] FP Romano et al., Anal Chem Oct. 2014 DOI: 10.1021/ ac503263h.

[10] SH Nowak et al., "Sub-pixel resolution with color X-ray camera Slcam," arXiv:1501.06825v1 (physics.ins-det) 2015.

[11] MN Boone et al., L. Nucl Instrum Methods Phys Res Sect. A 735 (2014) 644-48.

[12] I Reiche et al., Anal Chem 85 (2013) 5857-66.

[13] P Tack et al., Anal Chem 86 (2014) 8791-97.

\section{CAMBRIDGE}

\section{Scanning Electron Microscopy for the Life Sciences}

Heide Schatten

University of Missouri, Columbia

US\$120.00: Hb: 978-0-521-19599-7: $312 \mathrm{pp}$

Recent developments in scanning electron microscopy (SEM) have resulted in a wealth of new applications for cell and molecular biology, as well as related biological

New to the Advances in Microscopy and Microanalysis book series!

disciplines. It is now possible to analyze macromolecular complexes within their three-dimensional cellular microenvironment in near native states at high resolution, and to identify specific molecu les and their structural and molecular interactions. New approaches include cryo-SEM applications and environmental SEM (ESEM), staining techniques and processing applications combining embedding and resin-extraction for imaging with high resolution SEM, and advances in immuno-labeling. With chapters written by experts, this guide gives an overview of SEM and sample processing for SEM, and highlights several advances in cell and molecular biology that greatly benefited from using conventional, cryo, immuno, and high-resolution SEM.

\section{About the series}

The Press currently publishes the Microscopy and Microanalysis (MAM) journal in conjunction with the MSA, which reaches 4,000 microscopists and is affiliated with 12 international microscopy societies. The series would be a natural development from this journal, and will take a broad view of the discipline, covering topics from instrumentation to imaging, methodology and analysis across physical science, materials science, biology and medicine. Books commissioned for the series will range from advanced undergraduate textbooks through to research and practitioner oriented monographs for researchers. The series aims to produce a coherent source of material, encouraging the communication and exchange of ideas across these divergent fields, ensuring that the series appeals to a broad community in the physical and life sciences.

\section{Forthcoming titles in this series:}

Microscopic Nanocharacterization of Materials

by Michael Isaacson

Energy Filtered Electron Microscopy and Electron Spectroscopy by Richard Leapman

Dynamic Transmission Electron Microscopy

by Nigel Browning, Thomas LaGrange, Bryan Reed,

Henning Stahlberg, Bradley Siwick 